\title{
MEDIA COMPATIBLE PACKAGING AND ENVIRONMENTAL TESTING OF BARRIER COATING ENCAPSULATED SILICON PRESSURE SENSORS
}

\author{
David J. Monk, Theresa Maudie, Dennis Stanerson, John Wertz, \\ Gordon Bitko, Jeanene Matkin, and Slobodan Petrovic \\ Motorola, Semiconductor Products Sector, Sensor Products Division \\ Phoenix, AZ 85008
}

\begin{abstract}
Three specific examples of media exposure testing of pressure sensors with barrier coatings are presented. The examples are experiments used to simulate specific automotive and white goods applications. Experimentation was performed on an apparatus that was developed specifically for the exposure of these devices with in situ output voltage measurement in organic or aqueous environments. The first example illustrates the swelling of fluorosilicone gels in fuels and establishes a solubility parameter for one fluorosilicone gel between 6 and 8 $\left(\mathrm{cal} / \mathrm{cm}^{3}\right)^{1 / 2}$. While exposure to organic solutions has not been observed to cause catastophic failure of fluorosilicone gel filled devices, corrosion is accelerated in subsequent aqueous solution exposure. The second experiment was used to simulate automotive exhaust gases and water by exposing devices to a fuel mixture followed by an acidic solution. The third experiment was performed to study corrosion under parylene coatings during exposure to an alkaline test solution. This process has been modeled as a cathodic delamination phenomenon. Acceleration factor expressions have been estimated considering parylene coating thickness, solution $\mathrm{pH}$ and applied device supply voltage as acceleration means. These expressions have been used to evaluate parylene coated pressure sensors against a benchmark lifetime requirement: 2000 hours of exposure in a $\mathrm{NaOH} / \mathrm{NaHCO}_{3}$ buffer solution at $55^{\circ} \mathrm{C}$ with a $\mathrm{pH}$ of 11 and a supply voltage of $5 \mathrm{Vdc}$ applied continuously. For a $1 \%$ failure rate, parylene coated pressure sensors survived approximately 500 hours, whereas an alternative, multiple barrier coating survived over 2000 hours.
\end{abstract}

\section{INTRODUCTION}

Recently, questions about the viability of MEMS devices in the marketplace have begun to arise $[1,2]$. Lack of adequate infrastructure is considered a major limitation for the future success of MEMS products [3, 4]. A significant amount of infrastructure is necessary to develop sensors and actuators into high volume products, including: manufacturing; design, fabrication, packaging, testing, reliability and quality assurance facilities and personnel; marketing and applications; sales; management; etc. Analogies with the traditional IC industry can be used as guidelines for developing these functions; however, the uniqueness of MEMS devices often requires that these areas be addressed specifically for the device being produced. For example, this paper will propose a formal reliability method for characterizing media compatible pressure sensors. Reliability engineering techniques [5] are adapted to the media compatibility of pressure sensors by applying a physics-of-failure reliability approach [6]. This paper will: 1) provide motivation for addressing this problem, 2) identify the failure mechanisms observed, 3) show examples of using reliability engineering fundamentals to predict lifetime behavior for specific package types in specific media exposure experiments, 4) develop acceleration models for an observed failure mechanism, and 5) compare these results to a customer requirement.

Silicon pressure sensors have been offered by several companies since the late 1970s. A significant portion of the pressure sensor business has been specified for dry, noncorrosive gas environments or for short-term ( 24 - 48 hours), room temperature saline exposure environments for the medical industry. However, considerable opportunities are available for low-cost devices that can withstand long-term (i.e., several years) media compatible applications. To quantify acceptable long-term media compatibility, a general definition for media compatibility was formulated that can be applied to specific applications: the ability of a pressure sensor to perform its specified electromechanical function over an intended lifetime in the chemical, electrical, mechanical, and thermal environments encountered in a customer's application.

All sensors must provide this capability in at least dry, non-corrosive gas media. Specific markets that require additional media compatibility include automotive and/or white goods. Automotive compatible pressure sensors must survive fuel (i.e., organic) exposure in a temperature range from -50 to $150{ }^{\circ} \mathrm{C}$ for underhood applications. In addition, many are required to withstand exposure to salt water and/or strong acids (e.g., $\mathrm{NO}_{x}$ or $\mathrm{SO}_{x}$ and water), which form electrolytic aqueous solutions. Failure mechanisms are often different for exposure to these two classes of solutions.

White goods applications most often require exposure to aqueous solutions. For example, a washing machine application requires that the sensor is compatible with an alkaline solution bocause of the detergents added to tap water $[7,8]$. One current specification, for a customer who is interested in a water compatible pressure sensor for another white goods application, has been used as a benchmark for our development purposes. The exposure requirements are 2000 hours in a $\mathrm{NaOH} / \mathrm{NaHCO}_{3}$ buffered solution [9] with a pH of 11 at $55^{\circ} \mathrm{C}$.

There has been a significant amount of literature, especially patent literature, defining media compatible pressure sensor construction (e.g., [10-17]). Structures include backside exposure, designing the silicon to minimize exposure of the electronics, creating packages to minimize the exposure at crucial areas (including silicon-to-substrate bonding techniques), and the use of barrier coatings. Moreover, several companies sell media compatible silicon pressure sensors housed in stainless steel with a silicone oil transfer media and a stainless steel diaphragm. However, very little data exists for quantifying the media compatibility of a given device [12-14, 18, 19]. Some of this may be the result of the legality of publishing such data and some may be the result of the difficulty associated with performing such an experiment. Yet, analogous 
humidity experiments are the standard for quantifying reliability in IC devices. With this in mind, the goals for this work are: 1) to develop experimental test procedures for sensor exposure in these environments; 2) to determine the failure mechanisms during exposure testing and to model these results; and 3) to develop a product that has customer applicability.

\section{EXPERIMENTAL}

Our initial constraint in this work was to use existing silicon bulk micromachined, piezoresistive pressure sensors in the standard Motorola unibody package so that neither existing fabrication nor assembly manufacturing lines were impacted severly. Furthermore, low-cost, high-volume differential pressure sensor devices were required. Low-cost implies no more than approximately a $15 \%$ adder for media compability, and high-volume implies the capability of producing hundreds of thousands of devices each year.

Therefore, to provide media protection, barrier coatings were investigated initially. Specifically, fluorosilicone gels and/or parylene coatings have been used to produce these low cost devices. Several additional coatings were investigated, but they either did not satisfy the cost or the performance targets. Fluorosilicone gels and parylene coatings are significantly different materials, so a brief description of each is necessary.

Silicone gels are one or two part siloxanes that are drop dispensed and require a curing step [20-22]. Several vendors provide these materials, each with a slightly different formulation, and each with a slightly different curing mechanism. An example are the two-part fluorinated siloxanes that were used for these experiments. Often, these two-part materials consist of one part with a base polymer and a catalyst and a second part with the same base polymer, a multifunctional moiety, and an inhibitor. When the two parts are mixed and heated, the base polymer reacts (e.g., vinyl addition polymerization) with the multifunction species to form a gellike network. These materials are deposited in relatively thick coatings (on the order of millimeters); but because they are extremely low modulus materials, they have little effect on the device electrical performance.

Parylene C (poly(monochloro-para-xylylene)) coatings are deposited in a chemical vapor deposition chamber [23-25]. Dimer starting material is vaporized and then pyrolyzed to create a monomer gas that is drawn into a vacuum chamber containing the sensors. Because of the vacuum in the deposition chamber (approximately $80 \mathrm{mtorr}$ ), the material deposits and polymerizes in a very conformal manner. The nature of the nearroom-temperature deposition process allows for deposition on fully assembled devices with reasonably repeatable thicknesses. Thickness control is critical because parylene materials are much higher modulus materials than silicone gels, and they do produce a noticeable effect on electrical output. However, the repeatability of these shifts allows modeling of the effect of parylene on device parameters [26].

In the present work, experiments are presented to show the effects of organic solutions on gel coated devices, the effect of acidic solutions on gel coated devices, and the effect of alkaline solutions on parylene coated devices.

In the first experiment, various vendor supplied fluorosilicone gel materials used in coating pressure sensor packages were exposed to fuel solutions in a test apparatus that was designed as an automated organic solution exposure chamber (Fig. 1).

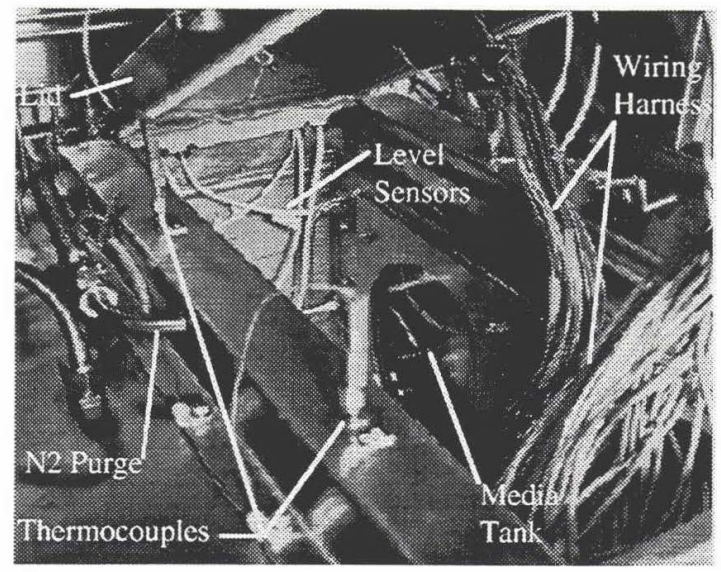

Figure 1. Organic solvent (fuel) media exposure system for monitoring pressure sensor offset in situ.

The test apparatus allows in situ offset (i.e., zero applied pressure output voltage) measurements with the ability to provide a supply voltage to the devices continuously. An automated mixing and circulating system is used to concoct various test solutions, often per SAE guidelines [27]. For this experiment reference fuel $\mathrm{C}$ with $5 \%$ corrosive water and fuel $\mathrm{C}$ with $20 \%$ ethanol and $5 \%$ corrosive water were evaluated. The corrosive water consists of $\mathrm{NaCl}, \mathrm{Na}_{2} \mathrm{SO}_{4}$, and $\mathrm{NaHCO}_{3}$ in $\mathrm{DI}$ water. This apparatus is equipped with an external reservoir for temperature control from approximately 10 to $90{ }^{\circ} \mathrm{C}$. Most importantly, considerable safety interlocks were designed into the system so that ultimately, if needed, the apparatus could shut itself off unattended. Visual and weight loss measurements were performed before and after these exposure experiments. In addition, manufacturing swelling data has been compared with solubility parameter information on the given solvents to provide an estimate for the solubility parameter of a representative gel [28].

In the second experiment, pressure sensors with various fluorogels as barrier coatings have been evaluated during exposure to fuel, acid, and combined fuel and acid solutions. The fuels and acids are known to react with the barrier materials [29]. Failure mechanisms expected during the evalution included swelling and dissolution of the polymer, and subsequent corrosion of any exposed metallization. Various fluorosilicone materials were evaluted for each test and lifetime statistics for the various tests computed.

In the final experiment, parylene coated pressure sensors have been used in alkaline exposure experiments. Corrosion under barrier coatings has been observed on several occasions to be the primary failure mode for devices when exposed to electrolytic aqueous solutions. Corrosion under parylene coatings in alkaline solutions was assumed to be analagous to the delamination of paint from metal surfaces and the subsequent corrosion of the metal (e.g., automobile bodies [30, 31]). The latter process has been modeled by diffusion of water through the polymer to the metal surface [31]. By solving Fick's First Law of Diffusion, with Ohm's Law as a boundary condition, a "delay time", t, prior to the onset of delamination can be established [31]: 


$$
t=\frac{\pi b^{2}}{4 D}\left(1-\frac{V}{4 R_{o} D F C_{o}}\right)
$$

where $b$ is the thickness of the coating, D is the diffusion coefficient of water through the coating, $\mathrm{V}$ is the applied voltage, $R_{0}$ is the resistivity of the coating, $F$ is Faraday's constant, and $C_{0}$ is the concentration of water at the surface of the coating. Our assumption was that delamination occurs prior to the onset of corrosion, similar to the model proposed by Leidheiser et al. (eqn. 1 and [31]); therefore, the corrosion failure times observed for these devices should be a function of coating thickness, applied bias, and solution concentration (eqn. 1). Unfortunately, we know very little about the delamination mechanism for parylene coatings in these environments, so we are assuming that the corrosion failure times are proportional to the delay times in the model proposed by Leidheiser et al. [31]. Therefore, the exposure experiment was designed to examine these variables by using a design of experiments technique [32]. Three parylene deposition runs were performed to create sets of devices with three different average parylene thicknesses. A modified Nanometrics Nanospec program was used to measure thickness. All fabrication, assembly, and surface preparation procedures remained constant within the existing manufacturing tolerances to minimize the effects of these variations on this experiment. Exposure of these devices was performed in individual exposure compartments (Fig. 2). All of the closed "cells" were placed in an oven at $55^{\circ} \mathrm{C}$. Continuous applied bias and offset measurements were performed during the 3022 hours of the experiment.

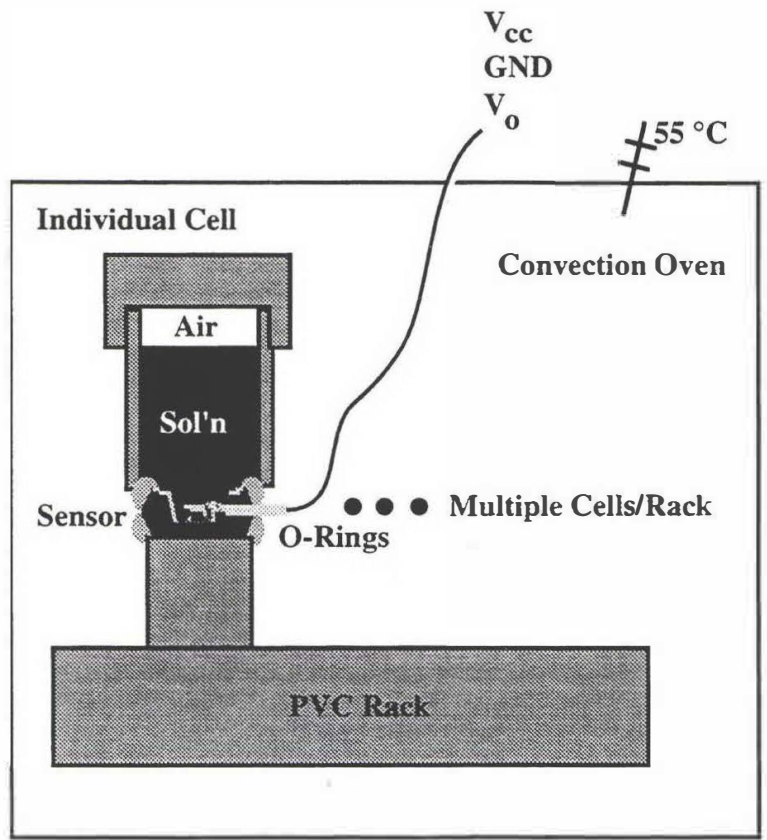

Figure 2. Individual compartments for exposure of barrier coated pressure sensors to aqueous solutions. A rack of 64 of these "cells" is placed into an oven at $55^{\circ} \mathrm{C}$.

\section{RESULTS AND DISCUSSION}

A more general discussion of the observed failure mechanisms with these type of devices is presented elsewhere [33]. Uniform, galvanic, and local corrosion; silicon etching; polymer swelling and/or dissolution; interfacial permeability (e.g., lead leakage [34] and/or adhesive delamination); and mechanical failures (i.e., cracking, creep. fatigue, etc.) all have been observed. In our experiments, polymer swelling and corrosion are observed and modeled in detail.

The swelling of fluorosilicone gels has been analyzed by attempting to determine the solubility parameter of a particular gel. Figure 3 shows a plot of manufacturer published swelling data plotted against the solubility parameter of the particular solvent [28]. The solubility parameter is a measure of the interaction energy between species:

$$
\delta_{i}=\left(\frac{\Delta E_{i}^{y}}{V_{i}}\right)^{1 / 2},
$$

where $\Delta E_{i}^{\nu}$ is the energy of vaporization and $\mathrm{V}_{i}$ the molar volume of the species. Because "like dissolves like", a peak in the curve represents the approximate solubility parameter of the polymer. Therefore, the solubility parameter for this particular fluorosilicone gel is between 6 and $8\left(\mathrm{cal} / \mathrm{cm}^{3}\right)^{0.5}$.

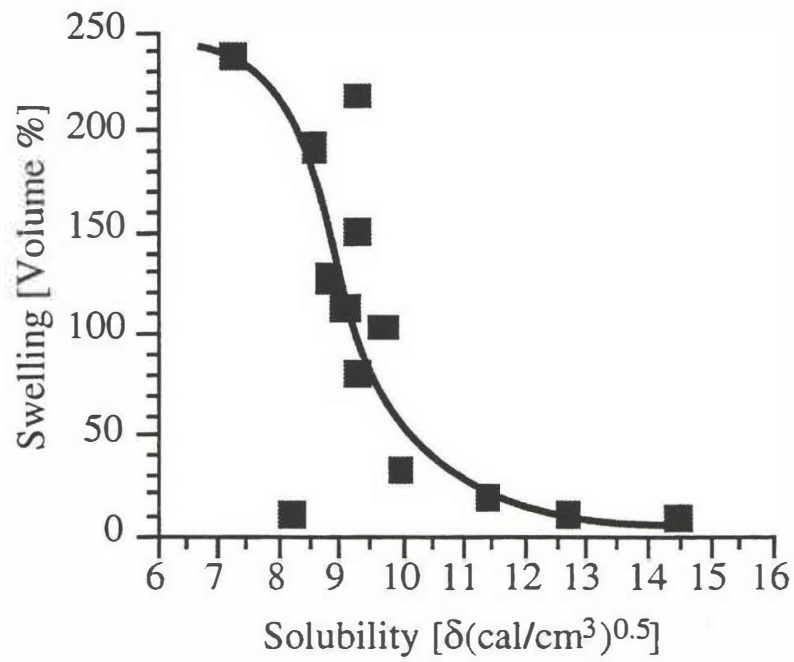

Figure 3. Swelling as a function of solubility parameter for a typical silicone gel using material supplier's swelling data following 8 days of exposure at $20{ }^{\circ} \mathrm{C}$ in various solvents and solubility parameters from ref. [35].

In Figure 4, the weight loss, assumed to be caused by loss of low molecular weight species into the solution, is shown for four fluorosilicone gels. The results show that the weight loss for a variety of fluorosilicone gels is not consistent. At least two potential causes for this result can be proposed. First, the extent of reaction of the gels varies, so certain gels have more low molecular weight species that will dissolve into the solution. Second, the solubility parameters of the polymers are different, resulting in a different degree of dissolution. Obviously, both of these can contribute to the final result. Regardless, each gel formulation must be treated as a separate material and 
experiments to understand these results more fully are being prepared. The most significant concern with this variability is the potential for subsequent corrosion that results from the exposure, especially if dissolution (i.e., weight loss) is great enough for metal regions to become exposed (e.g., Fig. 5).

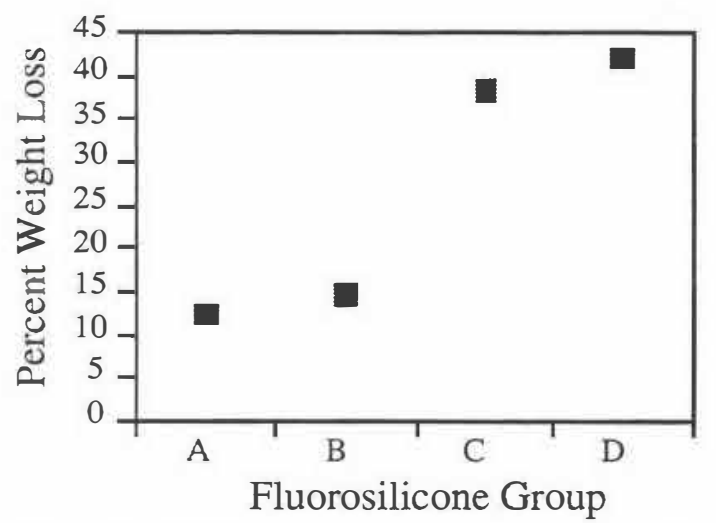

Figure 4. Weight loss measurements on four different fluorosilicone gels after 250 hours of exposure to reference fuel $C$ with $5 \%$ corrosive water at $65^{\circ} \mathrm{C}$.

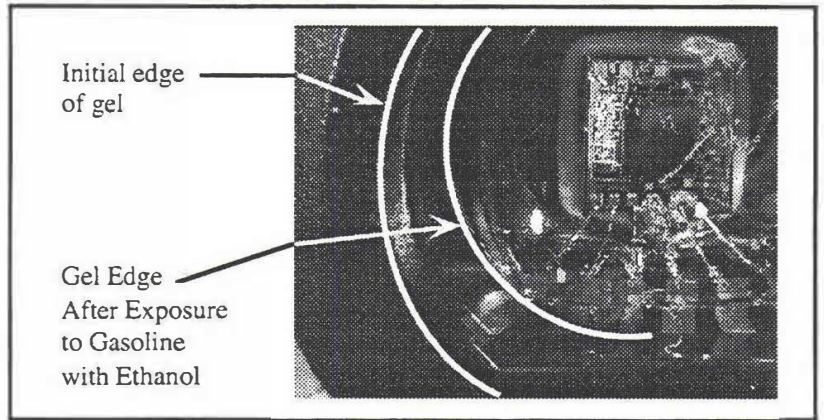

Figure 5. Photograph of a pressure sensor device after extended exposure to fuel containing corrosive water [27], followed by exposure to a strong acid. Evidence of the gel swelling during the test, and corresponding shrinkage after removal from the test media can be seen by the gel retracting away from the sidewall of the package.

The second experiment was performed to address this concern. Automotive applications require that sensors survive both organic solutions (e.g., fuels, oils, etc.) and aqueous solutions (e.g., salt water, $\mathrm{NO}_{\mathrm{x}}+$ water $=\mathrm{HNO}_{3}, \mathrm{SO}_{\mathrm{x}}+$ water $=$ $\mathrm{H}_{2} \mathrm{SO}_{4}$, etc.). In this experiment, fluorosilicone gel-filled devices were exposed to fuel ([27] for $250 \mathrm{hrs}$.) and then to nitric acid with a $\mathrm{pH}$ of 1.8 at $85{ }^{\circ} \mathrm{C}$, which is a standard specification to simulate under-the-hood operation. Failure lifetimes were recorded with in situ offset monitoring. For each group, this lifetime data was assumed to follow a Weibull cumulative failure distribution:

$$
R(t)=\exp \left[-\left(\frac{t}{\theta}\right)^{\beta}\right]
$$

Statistical likelihood analysis of the lifetime data was used to determine the $\theta$ factors (Table 1). These results can then be used to compare the performance of devices coated with different fluorosilicone gels. However, this technique can be plagued with the same limitations as traditional corrosion testing (i.e., the difficulty of simulating the field environment with a laboratory experiment) unless the environment is extremely well understood. As a supplier of pressure sensors, we are attempting to become as adept as possible at understanding customer applications and failure mechanisms. Assuming the test environment produces the same failure mechanism with similar kinetics, these experiments are an invaluable tool to compare media compatibility schemes and to estimate lifetime in a customer application provided the specifics of that application are known.

Table 1. Weibull Distribution Parameters for Fluorosilicone Gel Coated Pressure Sensors Exposed to Fuel and then to Nitric Acid (pH 1.8 at $85^{\circ} \mathrm{C}$ ).

\begin{tabular}{c|c|c}
\hline $\begin{array}{c}\text { Fluorosilicone } \\
\text { Group }\end{array}$ & $\begin{array}{c}\text { Characteristic Life, } \theta \\
\text { [hours] }\end{array}$ & $\mathrm{R}(\mathrm{t}=48$ hours $)$ \\
\hline A & 291 & $85 \%$ \\
B & 51 & $39 \%$ \\
C & 27 & $16 \%$ \\
D & 70 & $51 \%$ \\
\hline
\end{tabular}

This is the case in the final experimental example. The qualification procedure for a media compatible pressure sensor for a particular white goods customer has been estimated to be 2000 hours of exposure in a $\mathrm{NaOH} / \mathrm{NaHCO}_{3}$ buffered solution [9] with a $\mathrm{pH}$ of 11 at $55{ }^{\circ} \mathrm{C}$. Therefore, experiments with parylene coated sensors have been performed to 3022 hours with a continuous supply voltage and in situ offset monitoring in this environment. Again, failure distributions are modeled assuming a Weibull cumulative failure function. If all of the Weibull slope parameters are assumed to be 3 , indicating a "wear-out" failure mechanism (e.g., corrosion under a coating), the characteristic lifetimes can be modeled using analysis of variance to develop a linear expression that takes into account the effects of coating thickness, applied bias, and solution concentration (Fig. 6a):

$$
\begin{aligned}
\theta(h r s)= & (15000 \pm 5800)-(400 \pm 340) t_{p}[\mu m]+ \\
& (100 \pm 480) V_{c c}[V]-(1400 \pm 500) C_{o}[p H]- \\
& (40 \pm 16) V_{c c}^{2}\left[V^{2}\right]+(40 \pm 31) t_{p} \cdot C_{o}[\mu m \cdot p H]+ \\
& (50 \pm 33) V_{c c} \cdot C_{o}[V \cdot p H] \quad R^{2}=0.846
\end{aligned}
$$

In addition, these distributions can be used to obtain estimates of the $1 \%$ failure rate (Fig. 6b) or the failure rate at 2000 hours (Fig. 6c).

From these experimental results, the current parylene processing techniques do not produce a device that will satisfy the requirements of this customer. At a $\mathrm{pH}$ of 11 and a $5 \mathrm{~V}$ supply voltage, the characteristic lifetime (Fig. 6b) is approximately 450 hours for a $1 \%$ failure rate, which was assumed to be our constraint. Devices with fluorosilicone gel encapsulation alone performed similarly to the parylene encapsulated devices.

During these experiments, however, additional devices with a multiple organic barrier coating were observed to survive 3022 hours of exposure without failure. A subsequent experiment with 37 composite coatings with a "worst-case" manufacturing process have been exposed for 550 hours to $\mathrm{NaOH} / \mathrm{NaHCO}_{3}$ at $\mathrm{pH}$ of $12.5,55{ }^{\circ} \mathrm{C}$, and $15 \mathrm{Vdc}$ without 
incident. By using a conservative acceleration factor of $4 \mathrm{X}$ from equation 4 (or Fig. 6), this represents approximately 2200 hours of exposure in this environment. This estimate assumes that these devices fail with the same mechanism as the parylene encapsulated devices used to develop equation 4 .

Finally, additional techniques, to be presented elsewhere, are being developed to obtain a better understanding of why this combination of materials provides an enhanced barrier coating, and to determine if it still provides acceptable electrical performance. It is postulated that reduced amount of corrosive reactants and/or temperature at the corrosion sites prior to reaching the steady state may affect the results. In addition, perhaps the upper coating minimizes delamination of the of the lower coating, which is required before corrosion can begin. Further exposure testing is being performed to repeat these results and to better understand the barrier coating failure mechanism.

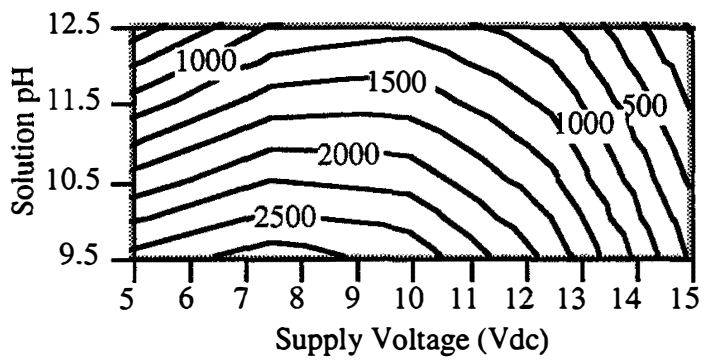

(a)

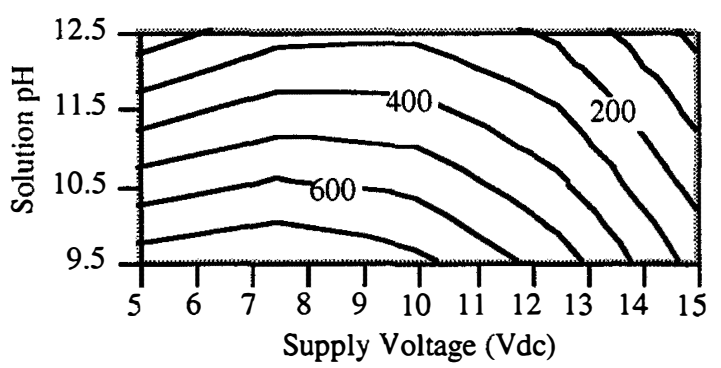

(b)

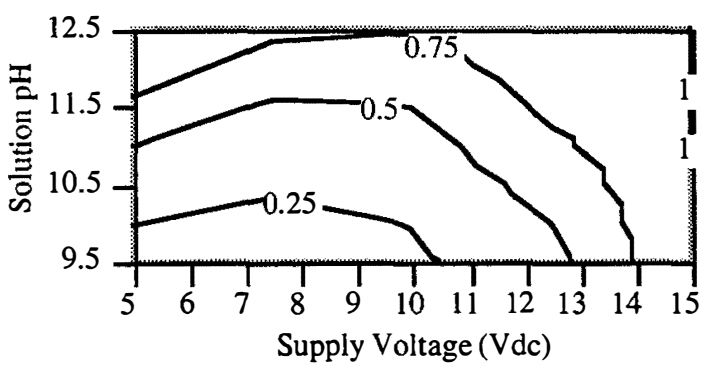

(c)

Figure 6. Failure analysis for $\approx 5 \mu \mathrm{m}$ parylene $C$ coated pressure sensors exposed to $\mathrm{NaOH} / \mathrm{NaHCO}_{3}$ at $55{ }^{\circ} \mathrm{C}$ with continuous supply voltage. (a) Weibull characteristic life, $\theta$, as a function of supply voltage and solution pH. (b) Characteristic lifetime for a failure rate of $1 \%$ as a function of supply voltage and solution pH. (c) Failure rate at 2000 hours as a function of supply voltage and solution $\mathrm{pH}$.

\section{CONCLUSIONS}

Several experimental test techniques have been developed for performing simulation of customer media applications. Separate organic (i.e., fuel, oil, etc.) and aqueous (i.e., acid, base, salt water, etc.) systems with continuous supply voltage, temperature control, in situ offset monitoring, and significant safety precautions have been developed. Complementary analytical techniques have been initiated to gain a better understading of the failure mechanisms. Failure mechanisms, including: corrosion, silicon etching, polymer swelling/dissolution, interfacial permeability, and mechanical failures have been observed with barrier coated silicon bulk micromachined, piezoresistive pressure sensors. The ultimate goal of this work is to develop an industry standard analogue to IC reliability for media compatible pressure sensors.

Three specific examples have been presented to illustrate the effects of polymer swelling/dissolution of polymer coatings in organic solutions and corrosion under polymer coatings in aqueous solutions. The polymer solubility parameter was used to begin to predict the swelling/dissolution failure mechanism. The corrosive failure mechanisms were being studied by analyzing failure distributions to allow comparison of media compatibility schemes and to benchmark development products with customer requirements. These environmental exposure simulation experiments require that the customer environment and the failure mechanism resulting from that requirement are very well understood. In the automotive environment, polymer swelling/dissolution from exposure to organic solutions (e.g., fuels) may not result in a catastrophic failure, but the subsequent acid exposure, used to simulate exhaust gases and water, has been observed to cause corrosive failures more rapidly than was observed on devices that have not been exposed to the organic solution.

It was also demonstrated that corrosion under parylene coatings in an alkaline environment for white goods applications is a function of polymer thickness, supply voltage, and solution concentration, as predicted by solving the Fickian diffusion problem for this type of system. This experiment showed that neither parylene nor gel coatings alone provided a barrier that was sufficient to satisfy all lifetime requirements. However, if it can be assumed that the same failure mechanism governs the failure of devices encapsulated with a multiple organic coating scheme, then the results indicated that a combination of barrier coatings will satisfy long term alkaline media exposure requirements.

\section{ACKNOWLEDGMENTS}

The authors are grateful to several members of Motorola Semiconductor Products Sector, including: the MEMS1 fabrication facility for providing die, the MKL and SPD Prototype assembly areas for packaging the die, the SPD Characterization Lab for testing devices, the Product Analysis Lab and Chemical and Surface Analysis Labs for helping with the analysis of failed devices, and the SPD Marketing group for helpful discussions concerning the prospects of developing a low-cost, high-volume, media compatible pressure sensor. 


\section{REFERENCES}

[1] F. Maseeh, "Lack of Manufacturing Know-How is Slowing the MEMS Revolution", Solid State Tech., 38, 50, (1995).

[2] F. Maseeh, "The Sensor-Based MEMS Market: An Underachiever?", Sensor Business News, 2, (1995).

[3] S. Horntvedt, "Industrialization of an R \& D Sensor Company", The 8th International Conference on Solid-State Sensors and Actuators, and Eurosensors IX, Stockholm, Sweden, (1995), 902.

[4] K. Petersen, "MEMS: What Lies Ahead?", The 8th International Conference on Solid-State Sensors and Actuators, and Eurosensors IX, Stockholm, Sweden, (1995), 894.

[5] K. C. Kapur and L. R. Lamberson, "Reliability Estimation: Weibull Distribution", in Reliability in Engineering Design. New York: John Wiley \& Sons, (1977), 291.

[6] J. M. Hu, "Physics-of-Failure-Based Reliability Qualification of Automotive Electronics", Communications in RMS, 1, 21, (1994).

[7] T. Maudie and J. Wertz, "Semiconductor Sensor Performance and Reliability for the Appliance Industry", International Appliance Technical Conference, Champaign/Urbana, IL, (1995),

[8] T. Maudie, "Improving Reliability of Electronic Appliance Pressure Sensors", Appliance, 39, (1995).

[9] D. R. Lide, "CRC Handbook of Chemistry and Physics", 75 ed. Boca Raton, FL: CRC Press, (1995), .

[10] A. D. Kurtz and J. R. Mallon, "Media Compatible Pressure Tranducer", U.S. Patent 4,222,277, September 16, 1980.

[11] W. P. Eaton and J. H. Smith, "A CMOS-Compatible, Surface Micromachined Pressure Sensor for Aqueous Ultrasonic Applications", Micromachining and Microfabrication '95, Austin, TX, SPIE (1995), 258.

[12] A. Hanneborg, M. Nese, and P. Øhlckers, "Silicon-toSilicon Anodic Bonding with a Borosilicate Glass Layer", $J$. Micromech. Microeng., 1, 139, (1991).

[13] A. Hanneborg, M. Nese, H. Jakobsen, and R. Holm, "Silicon-to-Thin Film Anodic Bonding", J. Micromech. Microeng., 2, 117, (1992).

[14] M. Nese and A. Hanneborg, "Anodic Bonding of Silicon to Silicon Wafers Coated with Aluminum, Silicon Oxide, Polysilicon or Silicon Nitride", Sensors and Actuators A, 37. 38, 61, (1993).

[15] M. Noble, "Environmental Concerns for Integrated Circuit Sensors", Measurement + Control, 19, 210, (1986).

[16] K. Ryan, J. Bryzek, and R. Grace, "Flexible Miniature Packaging from Lucas NovaSensor Provides Low-Cost Pressure Sensor Solutions to a Wide Variety of Hostile Media Applications", ECN, 118, (1995).

[17] S. Shoji, T. Nisase, M. Esashi, and T. Matsuo, "Fabrication of an Implantable Capacitive Type Pressure Sensor", The 4th International Conference on Solid-State Sensors and Actuators (Transducers '87), (1987), 305.

[18] T. Maudie, "Testing Requirements and Reliability Issues Encountered with Micromachined Structures", Proceedings of the Second International Symposium on Microstructures and Microfabricated Systems, Chicago, IL, ECS (1995), 223.

[19] A. Nakladal, K. Sager, and G. Gerlach, "Influences of Humidity and Moisture on the Long-Term Stability of Piezoresistive Pressure Sensors", Measurement, 16, 21, (1995).
[20] A. Polak, "Characterization and Use of Polyfluorosiloxanes in Automotive Applications", Materials Research Society Symposium Proceedings: Electronic Packaging Materials Science VI, San Francisco, CA, MRS (1992), 281.

[21] W. Fabianowski, D. Jaffe, and R. J. Jaccodine, "Mechanisms of Microelectronics Protection with Polysiloxane Polymers", 6th International SAMPE Electronics Conference, SAMPE (1992), 256.

[22] C. P. Wong, "Recent Advances in the Application of High Performance Siloxanes Polymers in Electronic Packaging”, 6th International SAMPE Electronics Conference, SAMPE (1992), 508.

[23] W. F. Beach, T. M. Austin, and R. Olson, "Parylene Coatings", in Electronic Materials Handbook ${ }^{\mathrm{TM}}$ : Volume 1:

Packaging, $1_{2}$ M. L. Minges, C. A. Dostal, and M. S. Woods, Eds. Materials Park, OH: ASM International, (1989), 789.

[24] W. F. Beach, C. Lee, D. R. Bassett, T. M. Austin, and R. Olson, "Xylylene Polymers", in Encyclopedia of Polymer Science and Engineering, 17, 2nd ed: John Wiley \& Sons, Inc., (1989), 990.

[25] W. F. Gorham, "A New, General Synthetic Method for the Preparation of Linear Poly-p-xylylenes", J. Polym. Sci., 4, 3027, (1966).

[26] D. J. Monk and M. Shah, "Thin Film Polymer Stress Measurement Using Piezoresistive Anisotropically Etched Pressure Sensors", Mat. Res. Soc. Symp. Proc., San Francisco, CA, MRS (1995), 103.

[27] S. C. R. Report, "Gasoline/Methanol Mixtures for Material Testing", Motorola Internal Report (SAE: 1990).

[28] F. Rodriguez, Principles of Polymer Systems, 2 ed. Washington: Hemisphere Publishing Corporation--McGrawHill Book Company, (1982).

[29] L. D. Fiedler, T. L. Knapp, A. W. Norris, and M. S. Virant, "Effect of Methanol/Gasoline Blends at Elevated Temperature on Fluorosilicone Elastomers", Society of Automotive Engineers International Congress and Exposition, SAE (1990).

[30] J. Leidheiser, H., "Mechanisms of De-adhesion of Organic Coatings from Metal Surfaces", Polymeric Materials for Corrosion Control, Chicago,IL, American Chemical Society, Washington,DC (1985), 125.

[31] W. Wang and J. Leidheiser, H., "A Model for the Quantitative Interpretation of Cathodic Delamination", Equilibrium Diagrams Localized Corrosion, ECS (1983), 255.

[32] D. C. Montgomery, Design and Analysis of Experiments, 3rd ed. New York: John Wiley \& Sons, (1991).

[33] T. Maudie, D. J. Monk, D. Zehrbach, and D. Stanerson, "Sensor Media Compatibility: Issues and Answers", Sensors Expo, Anaheim, CA, (1996).

[34] D. J. Monk, "Pressure Leakage through Material Interfaces in Pressure Sensor Packages", Sensors in Electronic Packaging, San Francisco, CA, ASME (1995), 87.

[35] E. A. Grulke, "Solubility Parameter Values", in Polymer

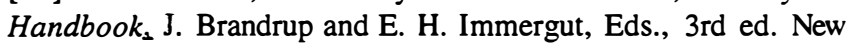
York: John Wiley \& Sons, (1989), VII/519. 